\title{
Enhancement DPT Method in Terms of Estimation Chirp Rate and Central Frequency Parameters of the LFM Signal
}

\author{
Nooshin RABIEE, Hamid AZAD, Naser PARHIZGAR \\ Dept. of Electrical Engineering, Shiraz Branch, Islamic Azad University, Shiraz, Iran \\ nooshin.rabiee@yahoo.com, azad@shirazu.ac.ir, parhizgar@iaushiraz.ac.ir
}

Submitted July 20, 2018 / Accepted December 7, 2018

\begin{abstract}
The discrete polynomial-phase transform (DPT) method estimates chirp rate and central frequency of LFM signal based on sequential estimation of polynomial phase parameters. This method uses Nonlinear Least Squares (NLS) technique (based on FFT) to estimate phase parameters of the LFM signal. Although NLS enjoys a high level of statistical accuracy, it entails high computational load. In this paper, in order to enhance the precision of estimation and also to reduce the computational load in DPT method, a technique called "combined" is proposed and used in DPT method in order to estimate chirp rate and central frequency of LFM signal. The combined technique firstly provides an initial estimate of frequency interval based on NLS criterion in single-exponential mode, then is using initial estimation and Random Basis Functions method (RBF). Simulation results are presented to demonstrate better performance of DPT method by combined technique in order to estimate phase parameters of LFM signal as compared with the existing techniques.
\end{abstract}

\section{Keywords}

Discrete Polynomial Phase Transform (DPT), Nonlinear Least Square (NLS), Synthetic Aperture Radar (SAR), Linear Frequency Modulation(LFM)

\section{Introduction}

The linear frequency modulation (LFM) signal is recognized with its two main parameters, the central frequency and the chirp-rate. Due to their widespread role in the frequency domain, LFM signals are of considerable use in radar [1], Sonar [2], ISAR [3], [4], communications [5], ultrasound [6] and geodesy [7] and many other areas.

The imaging of moving targets using synthetic aperture radar (SAR) is a specific application that also motivated the study of LFM signals. In order to form an output image in SAR, in all algorithms, it is necessary to estimate the azimuth direction parameters of the SAR, which include the Doppler centroid frequency and the azimuth FM rate [8]. Having accurate information about the phase pa- rameters of the LFM signal is necessary in the imaging of moving objects [9-11].The phase parameters of the LFM signal in all of the above applications contain important information and their estimation is very significant.

There are many techniques available to estimate the phase parameters of the LFM signal based on the maximum likelihood criterion (ML) [12]. The ML estimation of these parameters entails the optimization of a nonlinear cost function, which requires high computational load. ML [13] is in category of non-correlation algorithms. There are various suboptimal methods that are designed for the sequential estimation of the phase parameters with less computation. Among these techniques, there are the leastsquares technique [14], the technique of decreasing order [15], and the cyclostationary approach [16] [17]. Also, based on the discrete polynomial-phase transform (DPT), an effective technique has been developed by Peleg and Porat [18]. Among these different methods, the focus of this paper is on the DPT method.

The aim of this paper is enhancement of DPT method in order to estimate chirp rate and central frequency of LFM signal with high precision and to reduce computational load. The algorithms for sequential estimating of phase parameters reduce the order of polynomial in the exponent of complex exponential to a lower order polynomial. Using the frequency estimation techniques, the higher order parameter can be estimated. Then, by neutralizing the effect of the higher order parameter in the input data, it estimates the lower order parameter. This operation continues until all of the complex exponential parameters are estimated. In this study, a technique called "combined" is proposed and used in DPT method. Combined technique used NLS and RBF methods which are common methods for frequency estimation of a noisy complex exponential. It is also shown that the accuracy of the DPT method with combined technique is close to the Cramer-Rao lower bound (CRLB) as compared with the existing techniques and produces accurate estimation result.

The paper is organized as follows. In Sec. 2, the sequential estimation of phase parameters is described. The NLS and RBF methods, which both are used by the combined technique, are illustrated in Sec. 3. In Sec. 4, the 
proposed method is presented and the simulation results of DPT method based on NLS, RBF and combined technique (in the MATLAB software) are presented and compared in Sec. 5. The computational complexity of the above-mentioned methods is compared in Sec. 6. Finally, conclusions are given in Sec. 7 .

\section{Sequential Estimation of Phase Parameters}

The techniques developed in [15], [16] and [19] are based on sequential estimation of polynomial phase parameters. An LFM signal can be modeled as (1)

$$
\begin{gathered}
x(t)=a \exp \left[\mathrm{j}\left(2 \pi\left(0.5 k_{\mathrm{r}} t^{2}+f_{0} t\right)\right)\right], \\
y(t)=x(t)+n(t), \quad t=0, \ldots, N-1 .
\end{gathered}
$$

In this equation, $n(t)$ is the white Gaussian noise with zero mean and variance of $\sigma^{2}$. The received saturated signal-tonoise is $y(t)$. Chirp rate is $k_{\mathrm{r}}$ and central frequency is $f_{0}$. The objective is to estimate $k_{\mathrm{r}}$ and $f_{0}$ from available finite and noisy samples. Having the parameters $k_{\mathrm{r}}$ and $f_{0}$, the amplitude $a$ can be estimated directly, which is due to the linear relationship between the signal and its amplitude. The algorithms for sequential estimating of phase parameters reduce the order of polynomial in the exponent of complex exponential to a lower order polynomial. Using the frequency estimation techniques, the higher order parameter can be estimated. Then, by neutralizing the effect of the higher order parameter in the input data, it estimates the lower order parameter. This operation continues until all of the complex exponential parameters are estimated. If the noise-free signal is defined as (1), we can define $r(t)$ as follows:

$$
\begin{array}{r}
r(t)=x(t+\tau) x^{*}(t)=A \exp \left[\mathrm{j} 2 \pi\left(2 \cdot 0.5 \cdot t \cdot k_{\mathrm{r}} \cdot \tau\right)\right], \\
t=0, \ldots, N-1-\tau .
\end{array}
$$

In this equation, the complex amplitude $A$ is defined as $|a|^{2} \exp \left[\mathrm{j}\left(f_{0} \tau+k_{\mathrm{r}} \tau^{2}\right)\right] . \tau$ is a positive integer and the sign star $(*)$ represents the conjugate of the complex operator. With respect to (3), it can be seen that the signal $r(t)$ is a sinusoidal signal with frequency of $\tau k_{\mathrm{r}}$. And transform $r(t)$ is similar in form to the one developed in [19].

Therefore, it is possible to estimate $k_{\mathrm{r}}$ using frequency estimation methods and following that the demodulated signal of $x(t)$ can be considered as follows:

$$
\begin{aligned}
z(t) & =x(t) \exp \left[-\mathrm{j} 2 \pi\left(0.5 \widehat{k_{r}} t^{2}\right)\right] \\
& \simeq a \exp \left(\mathrm{j} 2 \pi f_{0} t\right) .
\end{aligned}
$$

In (4), $\hat{k}_{\mathrm{r}}$ represents the estimation of chirp rate. In the case of $\hat{k_{\mathrm{r}}}=k_{\mathrm{r}}$, we can obtain the parameter $f_{0}$ using the generated signal $z(t)$ and a frequency estimation method. If the parameter $k_{\mathrm{r}}$ is estimated with an error, this error will also affect the estimation of $f_{0}$.

\section{Nonlinear Least Squares (NLS) and Random Basis Function (RBF) Methods}

\subsection{Random Basis Functions (RBF)}

The RBF method attempts to match the input data with a linear combination of basis functions. The basis functions have non-linear parameters. The RBF method can also be extended when we have some non-linear functions [20]. It is assumed that $y(t), t=0, \ldots, N-1$ is the received data and the basis function that we want to fit the data with is

$$
s[t]=A \varnothing[t ; \theta], \quad t=0,1, \ldots, N-1
$$

in which $A$ is the complex amplitude and $\varnothing[t ; \boldsymbol{\theta}]$ is the basis functions, which is a function of non-linear and unknown parameter of $\boldsymbol{\theta}=\left[\theta_{1}, \theta_{2}, \ldots, \theta_{p}\right]^{\mathrm{T}}$. In this method, to estimate the frequency $f_{0}$ of a complex sinusoidal, the function $s[t]$ will be as following:

$$
s[t]=A \exp \left(\mathrm{j} 2 \pi f_{0} t+\varphi\right)
$$

where $A$ is the unknown complex amplitude, $\theta=\left[f_{0}\right]$ and $\varphi$ is the initial phase. By minimizing (7), $A$ and $f_{0}$ are computed:

$$
J\left(A, f_{0}\right)=\sum_{n=0}^{N-1}\left|y[t]-A \exp \left(\mathrm{j} 2 \pi f_{0} t+\varphi\right)\right|^{2} .
$$

If a complex sine is embedded in Gaussian white complex noise, the NLS estimator used in (7) is equivalent to the maximum likelihood estimator (ML) [21]. The ML estimator firstly minimizes (7) on the amplitude and then on the frequency $f_{0}$. When we have a complex exponential, the process of estimating the frequency using ML estimator will be equal to finding the frequency at which the periodogram is maximized (which is computed by FFT) [22]. In this estimation the data and basis functions are considered to be complex. To perform the minimization of (7) optimally, we first start by minimizing the following expression:

$$
J\left(A_{1}, A_{2}\right)=E_{\theta}\left[\sum_{t=0}^{N-1}\left|y[t]-A_{1} \varnothing\left[t ; \Theta_{1}\right]-A_{2} \varnothing\left[t ; \Theta_{2}\right]\right|^{2}\right] .
$$

Assuming that the unknown parameter $\Theta$ is in the interval $[-a, a]$. Thus, the interval for the parameter $\Theta$ is divided into two equal intervals. The probability density function for $\Theta_{1}$ is assumed to be a uniform distribution in the interval $[-a, 0]$ and the uniform distribution of $\Theta_{2}$ for the interval $[0, a]$. Then, using the minimization of (8), we obtain the estimates for the amplitudes $A_{1}$ and $A_{2}$. Through computing the power associated with the amplitude of each interval, the interval with higher power is selected as a new interval. Again, we divide the selected frequency interval into two equal intervals and obtain the estimated power of the two new intervals. We select the more powerful one as a new interval in which the nonlinear parameter is availa- 
ble. After repeating the above steps, regarding the expected accuracy, we consider the complex exponential frequency equal to the value in the middle of the last interval.

Repetitions are guaranteed to converge to the estimated frequency, it is because of this fact that in each step the frequency interval at which the nonlinear parameter exists, is halved. The estimation of the two amplitudes $A_{1}$ and $A_{2}$ is done by simply estimating the least squares in each step. The reason for this is that amplitudes are linear functions. In the estimation of $A_{1}$ and $A_{2}$, which minimizes (8), the data vectors, amplitude, and estimated parameter are defined as follows:

$$
\begin{aligned}
\mathbf{y} & =[y(0), y(1), \ldots, y(N-1)]^{\mathrm{T}}, \\
\mathbf{a} & =\left[A_{1}, A_{2}\right]^{\mathrm{T}}, \\
\boldsymbol{\Theta} & =\left[\Theta_{1}, \Theta_{2}\right]^{\mathrm{T}}, \\
\mathbf{H}(\Theta) & =\left[\begin{array}{cr}
\varnothing\left[0: \Theta_{1}\right] & \varnothing\left[0: \Theta_{2}\right] \\
\varnothing\left[1: \Theta_{1}\right] & \varnothing\left[1: \Theta_{2}\right] \\
\vdots & \vdots \\
\varnothing\left[N-1: \Theta_{1}\right] & \varnothing\left[N-1: \Theta_{2}\right]
\end{array}\right] .
\end{aligned}
$$

$\mathbf{H}(\Theta)$ is a random and complex matrix and its dimension is $N \times 2$. With this definition, equation (9) is obtained as follows:

$$
J(\mathbf{A})=E_{\theta}\left[(\mathbf{y}-\mathbf{H}(\theta) \mathbf{a})^{\mathrm{H}}(\mathbf{y}-\mathbf{H}(\theta) \mathbf{a})\right] .
$$

In order to compute the amplitude $a$, using complex derivative property, we have:

$$
\begin{aligned}
\frac{\partial \mathrm{J}(\mathbf{a})}{\partial \mathbf{a}} & =E_{\theta}\left[\frac{\partial}{\partial \mathbf{a}}(\mathbf{y}-\mathbf{H}(\theta) \mathbf{a})^{\mathrm{H}}(\mathbf{y}-\mathbf{H}(\theta) \mathbf{a})\right] \\
& =E_{\theta}\left[\frac{\partial}{\partial \mathbf{a}}\left(-\boldsymbol{H}^{\mathrm{H}}(\theta)\right)(\mathbf{y}-\mathbf{H}(\theta) \mathbf{a})^{*}\right]=0 .
\end{aligned}
$$

After solving (11) we have:

$$
E_{\theta}\left[\mathbf{H}^{\mathrm{H}}(\theta) \mathbf{H}(\theta)\right] \hat{\mathbf{a}}=E_{\theta}^{\mathrm{H}}[\mathbf{H}(\theta)] \mathbf{y} .
$$

With respect to the right-hand side of (12) from (9) we have

$$
\mathrm{E}_{\theta}[\mathbf{H}(\Theta)]=\left[\begin{array}{cr}
E\left[\varnothing\left[0: \Theta_{1}\right]\right] & E\left[\varnothing\left[0: \Theta_{2}\right]\right] \\
E\left[\varnothing\left[1: \Theta_{1}\right]\right] & E\left[\varnothing\left[1: \Theta_{2}\right]\right] \\
\vdots & \vdots \\
E\left[\varnothing\left[N-1: \Theta_{1}\right]\right] & E\left[\varnothing\left[N-1: \Theta_{2}\right]\right]
\end{array}\right] .
$$

It is also assumed that $\Theta_{1}$ and $\Theta_{2}$ are independent of each other. In the complex exponential form, we have $\Theta=F_{0}, \varnothing\left[t ; \Theta_{1}\right]=\exp \left(\mathrm{j} 2 \pi F_{0} t+\varphi\right), \mathrm{E}\left[\left|\varnothing\left[t ; \Theta_{1}\right]\right|^{2}\right]=1$. The frequency in the RBF method is considered to be between
-0.5 and 0.5 . In order to find $\mathrm{E}[\varnothing[t ; \Theta]]$, we use (13) and (14) and consider a uniform PDF for $F_{0}$ in the frequency interval of $\left[f_{1}, f_{2}\right]$ that gives us (14) and (15):

$$
\begin{aligned}
& E_{\theta}\left[\mathbf{H}^{\mathrm{H}}(\theta) \mathbf{H}(\theta)\right]= \\
& {\left[\begin{array}{cc}
\sum_{t=0}^{N} E\left[\left|\varnothing\left[t ; \Theta_{1}\right]\right|^{2}\right] & \sum_{t=0}^{N} E\left[\varnothing^{*}\left[t ; \Theta_{1}\right] \varnothing\left[t ; \Theta_{2}\right]\right] \\
\sum_{t=0}^{N} E\left[\varnothing\left[t ; \Theta_{1}\right] \varnothing^{*}\left[t ; \Theta_{2}\right]\right] & \sum_{t=0}^{N} E\left[\left|\varnothing\left[t ; \Theta_{2}\right]\right|^{2}\right]
\end{array}\right] .} \\
& E[\varnothing[t ; \Theta]]=E\left[\varnothing\left[t ; F_{0}\right]\right]=E\left[\exp \left(\mathrm{j} 2 \pi F_{0} t+\varphi\right)\right]= \\
& \int_{f_{1}}^{f_{2}} \exp \left(\mathrm{j} 2 \pi F_{0} t+\varphi\right) P_{F_{0}}\left(f_{0}\right) \mathrm{d} f_{0}= \\
& \int_{f_{1}}^{f_{2}} \exp \left(\mathrm{j} 2 \pi F_{0} t+\varphi\right) \frac{1}{f_{2}-f_{1}} \mathrm{~d} f_{0}=\frac{\sin (\pi B t)}{\pi B n} \exp (\mathrm{j} 2 \pi \mu t+\varphi)
\end{aligned}
$$

in which $B=f_{2}-f_{1}$ and $\mu=\left(f_{2}+f_{1}\right) / 2$ are the bandwidth and center of the frequency interval, respectively. Using (12), (14) and (15), equation (16) can be achieved. In this equation, $\omega_{\mathrm{s}}^{(1)}[t]=\sin \left(\pi B^{(1)} t\right) /\left(\pi B^{(1)} t\right)$.

$\left[\begin{array}{l}\hat{A}_{1} \\ \hat{A}_{2}\end{array}\right]=$

$$
\begin{aligned}
& {\left[\begin{array}{cc}
N & \sum_{t=0}^{N-1} \omega_{\mathrm{s}}^{2}[t] \exp \left[-\mathrm{j} 2 \pi\left(\mu_{1}^{(1)}-\mu_{2}^{(1)}\right) t\right] \\
\sum_{t=0}^{N-1} \omega_{\mathrm{s}}^{2}[t] \exp \left[\mathrm{j} 2 \pi\left(\mu_{1}^{(1)}-\mu_{2}^{(1)}\right) t\right] & N
\end{array}\right]^{-1}} \\
& \times\left[\begin{array}{l}
\sum_{t=0}^{N-1} y[t] \omega_{\mathrm{s}}[t] \exp \left[-\mathrm{j} 2 \pi \mu_{1}^{(1)} t+\varphi\right] \\
\sum_{t=0}^{N-1} y[t] \omega_{\mathrm{s}}[t] \exp \left[-\mathrm{j} 2 \pi \mu_{2}^{(1)} t+\varphi\right]
\end{array}\right] .
\end{aligned}
$$

In [23], by matching available data and complex exponential functions with random frequency and by performing below steps, the amount of complex exponential frequency is estimated. For estimation of a complex sine in noise, the following stages can be considered:

For the first step, from $k$ steps, which is shown with the superscript (1), the first frequency interval $f_{1_{1}}^{(1)}=-0.5$, $f_{1_{\mathrm{u}}}{ }^{(1)}=0$, and the second interval $f_{21}{ }^{(1)}=0, f_{2 \mathrm{u}}{ }^{(1)}=0.5$ is chosen. The center of the first and second frequency intervals is equal to $\mu_{1}=-1 / 4$ and $\mu_{2}=1 / 4$ and the frequency bandwidth is chosen to be $B^{(1)}=0.5$.

In Step 2 by using (16), we estimate the complex amplitude of two intervals chosen in the previous step. In Step 3, from the previous two intervals, we choose the interval with higher power $\left|A^{(1)}\right|^{2} \cdot\left|A^{(1)}\right|^{2}$, and then divide it into two separate intervals. In this stage, $f_{21}{ }^{(2)}, f_{2_{u}}{ }^{(2)}, f_{1_{1}}{ }^{(2)}$, $f_{1_{u}}{ }^{(2)}, \mu_{1}{ }^{(2)}, \mu_{2}{ }^{(2)}, B^{(2)}=B^{(1)} / 2=1 / 4$ must be ascertained. When we compare the absolute value of the two amplitudes, the effect of $\varphi$ is removed and knowing its 
value is not important anymore. Thus, an RBF estimator can be used for any signal with an arbitrary initial phase. In Step 4 based on the third steps' estimated interval and according to the upper and lower limits of the new interval, we repeat the first and second steps. This will continue as long as the bandwidth is narrowed enough. Notice that in each step, the bandwidth decreases to $1 / 2$ of the previous step and therefore the bandwidth of the last interval is $1 / 2^{k}$.

And in Step 5, after repeating the steps for $k$ times, the estimated frequency is equal to the midpoint of the last frequency interval, or in other words:

$$
\hat{f}_{0}=\frac{1}{2}\left(f_{\mathrm{u}}^{(k)}+f_{1}^{(k)}\right) .
$$

\subsection{Nonlinear Least Squares Method}

In general, NLS is a criterion in which the parameters of the signal model are chosen in such a way that the sum of squares for the difference between the model and the data is minimized. Following that, this criterion considers the amount of selected parameters as the estimation of signal parameters. This criterion is also used in estimating complex single or multi exponential parameters in noise. Suppose that the signal model is obtained from the sum of several complex exponentials at time $t$, it is defined as:

$$
x(t)=\sum_{k=1}^{p} \alpha_{k} \exp \left[\mathrm{j}\left(\omega_{k} t+\varphi_{k}\right)\right] .
$$

$x(t)$ is equal to the sum of $p$ noise-free complex exponentials. Here $\alpha_{k}$ is the amplitude, $\omega_{k}$ is the angular frequency and $\varphi_{k}$ is the initial phase of the $k$-th complex exponential. The value of $\omega_{k}$ is in the interval $[-\pi, \pi], \varphi_{k}$ is a non-random phase in the interval $[-\pi, \pi]$ and $\alpha_{k}>0$. Also, $y(t)$ is the input data in time $t$ which is obtained from saturation of $x(t)$ with $n(t)$ noise.

$$
y(t)=x(t)+n(t) .
$$

To estimate the complex exponential parameters, we must minimize the function $f$ in (20) based on the unknown parameters of the problem:

$$
f(\omega, a)=\sum_{t=0}^{N-1}|y(t)-x(t)|^{2} .
$$

In the case that $f$ is a nonlinear function of $\{\omega, \varphi\}$, the method that determines the unknown parameters by minimizing (20) is called the nonlinear least squares (NLS) method. Equation (20) depends on the three parameters $\left\{\alpha_{k}\right\},\left\{\varphi_{k}\right\}$, and also $\left\{\omega_{k}\right\}$. We can write the function $f$ in (20) as the vectorial form of (21) to (25):

$$
\begin{gathered}
A_{K}=a_{k} \exp \left(\mathrm{j} \varphi_{k}\right), \\
\mathbf{a}=\left[A_{1}, \ldots, A_{p}\right]^{\mathrm{T}}, \\
\mathbf{y}=[y(0), \ldots, y(N-1)]^{\mathrm{T}},
\end{gathered}
$$

$$
\begin{gathered}
\mathbf{B}=\left[\begin{array}{ccc}
1 & \cdots & 1 \\
\vdots & \ddots & \vdots \\
\exp \left(\mathrm{j} N \omega_{1}\right) & \cdots & \exp \left(\mathrm{j} N \omega_{p}\right)
\end{array}\right], \\
f=[\mathbf{y}-\mathbf{a B}]^{\mathrm{H}}[\mathbf{y}-\mathbf{a B}] .
\end{gathered}
$$

Equation (25) is the vectorial form of (20) in which the matrix $\mathbf{B}$ is a Vandermonde matrix, and if $p<N$, is full rank. The number of complex exponentials is $p$ and the number of the input data is $N$. With the above definitions, we can write (25) as (26):

$$
\begin{aligned}
f= & {\left[\mathbf{a}-\left(\mathbf{B}^{\mathrm{H}} \mathbf{B}\right)^{-1} \mathbf{B}^{\mathrm{H}} \mathbf{y}\right]^{\mathrm{H}}\left[\mathbf{B}^{\mathrm{H}} \mathbf{B}\right]\left[\mathbf{a}-\left(\mathbf{B}^{\mathrm{H}} \mathbf{B}\right)^{-1} \mathbf{B}^{\mathrm{H}} \mathbf{y}\right]+} \\
& \mathbf{y}^{\mathrm{H}} \mathbf{y}-\mathbf{y}^{\mathrm{H}} \mathbf{B}\left(\mathbf{B}^{\mathrm{H}} \mathbf{B}\right)^{-1} \mathbf{B}^{\mathrm{H}} \mathbf{y} .
\end{aligned}
$$

In order to minimize equation $f$, the value of $\omega$ must be chosen in such a way that maximizes the third term in (26):

$$
\begin{gathered}
\hat{\omega}=\arg \max _{\omega}\left[\mathbf{y}^{\mathrm{H}} \mathbf{B}\left(\mathbf{B}^{\mathrm{H}} \mathbf{B}\right)^{-1} \mathbf{B}^{\mathrm{H}} \mathbf{y}\right], \\
\hat{a}=\left.\left(\mathbf{B}^{\mathrm{H}} \mathbf{B}\right)^{-1} \mathbf{B}^{\mathrm{H}} \mathbf{y}\right|_{\omega=\hat{\omega}} .
\end{gathered}
$$

In the case that input signal includes a complex exponential, according to the NLS general solution in (27) and (28), the frequency estimation will be as follows:

$$
\begin{aligned}
\hat{\Phi}_{p}(\omega) & =\left|\sum_{t=0}^{N-1} y(t) \exp (-\mathrm{j} \omega t)\right|^{2}, \\
\hat{\omega} & =\arg \max _{\omega} \hat{\Phi}_{p}(\omega) .
\end{aligned}
$$

According to (29) and (30), the estimated frequency equal to the value that maximize $\Phi(\omega)$. Also, the term inside the absolute value sign in (29) can be expanded using Fast Fourier Transform (FFT). Equations (29) and (30) represent a nonparametric method known as periodogram [24].

\section{Proposed Method}

In the DPT method, in order to estimate the parameters of the LFM signal, $r(t)$ and $z(t)$ are formed to estimate the chirp rate and the central frequency, respectively. $Z(t)$ and $r(t)$ are samples of complex exponentials with frequency corresponding to these parameters. Then, by using the NLS method, in the special case of single-exponential mode, we estimate these two parameters. In the single-exponential mode, the NLS method is exactly equal to the periodogram method. The periodogram method that uses FFT to reduce the computations, have some problems: 1) the MSE of the estimated frequency is limited due to the frequency bin errors over the constant length of FFT. 2) The high rate of computation in this method. 
In this paper, in order to enhance performance of DPT method in terms of estimation chirp rate and central frequency of the LFM signal, we propose and use combined technique. Like the RBF method, combined technique seeks the estimation of complex exponential frequency with an acceptable accuracy but with less computation compared to NLS in single-exponential mode. The main problem with the RBF method is related to low SNRs, which is related to the minimum inaccurate estimation of an interval of a step (among $k$ steps) which is caused by the low signal power-to-noise power ratio. In this condition, the estimator will be in the wrong interval and consequently the estimated frequency would be wrong. In the combined technique, first, by using (31) and (32), initial estimation from frequency interval in which there is complex exponential, is obtained. Then by using initial estimated frequency interval and RBF method, complex exponential frequency will be estimated.

$$
\begin{gathered}
\hat{f}_{\mathrm{ML}}=\arg \max _{(\lambda)}\{Y(\lambda)\}, \\
Y(\lambda)=\left|\sum_{t=0}^{N-1} y(t) \exp (-\mathrm{j} 2 \pi t \lambda)\right|^{2} .
\end{gathered}
$$

We show the FFT length used to compute (32) with $N_{\text {FFT-syn. }}$. The value of $N_{\text {FFT-syn }}$ is chosen to be small because of this fact that we use periodogram only to achieve an initial estimation of the complex exponential frequency, and also, due to this fact that in order to avoid extra computation, it is not necessary to use a large FFT length. If $f_{\mathrm{d}}$ is the estimated frequency using periodogram, the considered interval for estimating the frequency based on the RBF method is:

$$
\left(f_{\mathrm{d}}-\frac{1}{2 N_{\text {FFt__yn }}}, f_{\mathrm{d}}+\frac{1}{2 N_{\text {FFt_syn }}}\right) .
$$

In RBF method, when two sub-frequency intervals are small, the error associated with the interval selection decreases due to the reduction of the noise power. According to the estimated interval and equation (16), we compute the amplitudes of two sub-intervals resulted from the main interval in (33). In the next stage, each sub-interval which has higher power will be considered as the new interval, and these steps will be repeated like the RBF method. Simulation results of the aforementioned methods will be presented in the next section.

\section{Simulation Results}

Simulation results illustrate and compare performance of DPT method with NLS, RBF and Combined techniques in terms of estimation chirp rate and central frequency of the LFM signal. The performance is compared with CRLB inverse as shown in [18]. In Fig. 1 and 2, while the central frequency is 0.2 and chirp rate is 0.1 , the performance of the DPT method for the LFM signal is shown. The number of input samples is 20 and the number of independent tests to compute MSE inverse for each value of SNR is 100,000 . The FFT length for the NLS method is 256. Figure 1 shows MSE inverse and CRLB inverse in DPT method for estimating the chirp rate of the LFM signal, while, the SNR of the input signal changes. Figure 2 shows the changes of MSE inverse values for estimating the central frequency of the LFM signal. In the DPT method, we have the estimation of the chirp rate by using the NLS method. As can be seen in Fig. 1 and 2, MSE inverse values in the DPT method reach to a constant value after a specific SNR and we will have an increasing difference between MSE inverse and CRLB inverse in higher values of SNR, which cause a gap between MSE inverse and CRLB inverse. The reason to this issue is the NLS estimation problem with single complex exponentials (periodogram based on FFT). In Fig. 2, because of the chirp rate estimation error, MSE inverse values in central frequency estimation don't converge to CRLB inverse.

In Fig. 3, for two methods (DPT with NLS and DPT with RBF), MSE inverse versus SNR of the chirp rate estimation for a signal with the chirp rate of 0.1 and the central frequency of 0.2 can be seen. According to this figure, in DPT with RBF method, the steps of RBF method is 15 and

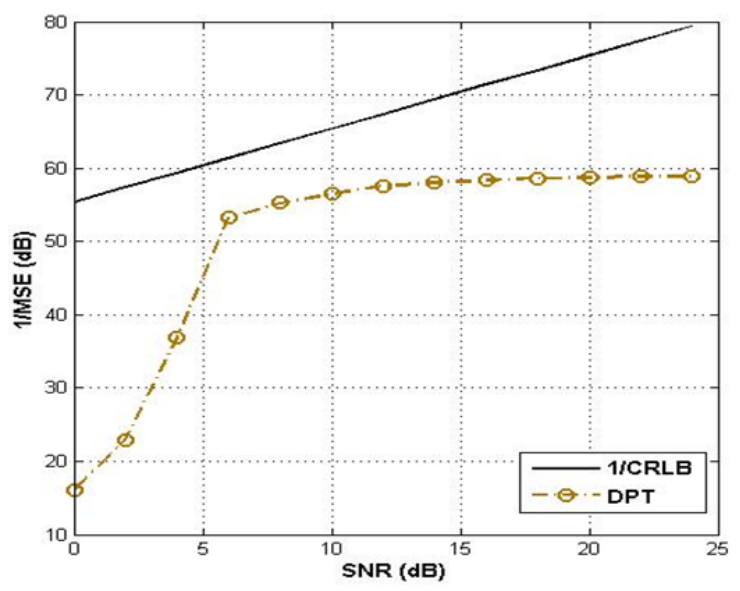

Fig. 1. MSE inverse versus SNR, for chirp rate estimation of the LFM signal in DPT method $\left(k_{\mathrm{r}}=0.1, f_{0}=0.2\right)$

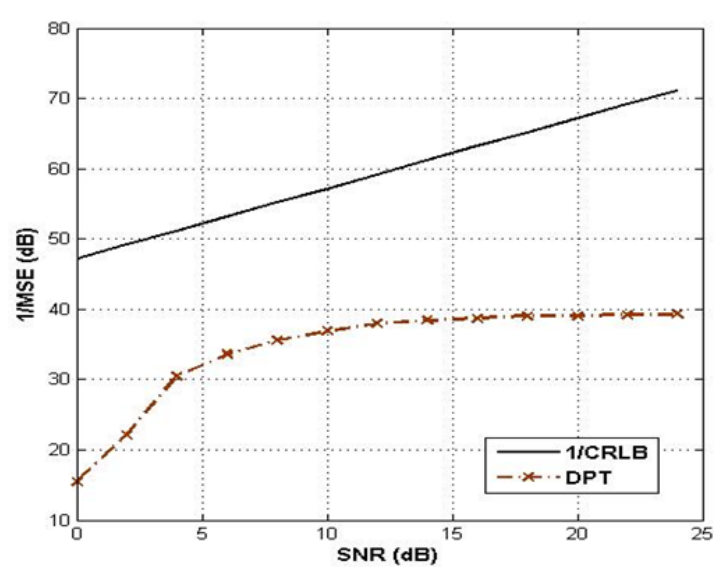

Fig. 2. MSE inverse versus SNR, for central frequency estimation of the LFM signal in DPT method $\left(k_{\mathrm{r}}=0.1\right.$, $\left.f_{0}=0.2\right)$. 


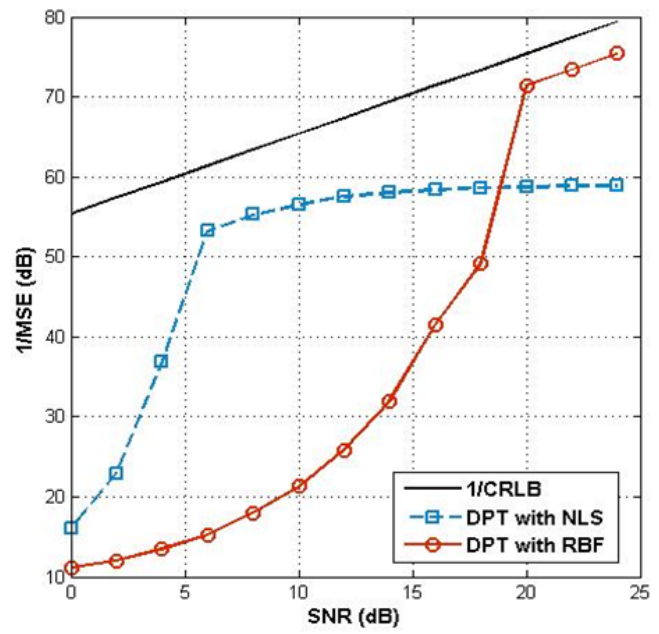

Fig. 3. MSE inverse versus SNR for chirp rate estimation of the LFM signal, using DPT with NLS and DPT with RBF methods.

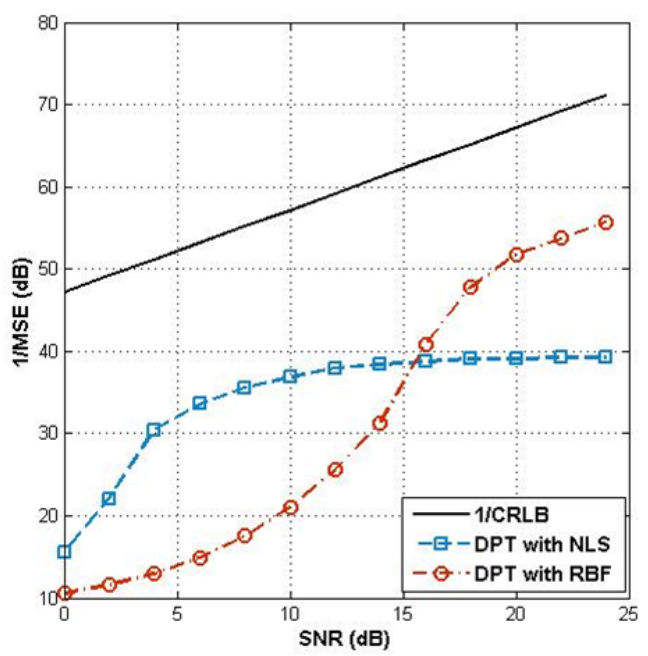

Fig. 4. MSE inverse versus SNR for central frequency estimation of the LFM signal, using DPT and NLS and DPT with RBF methods.

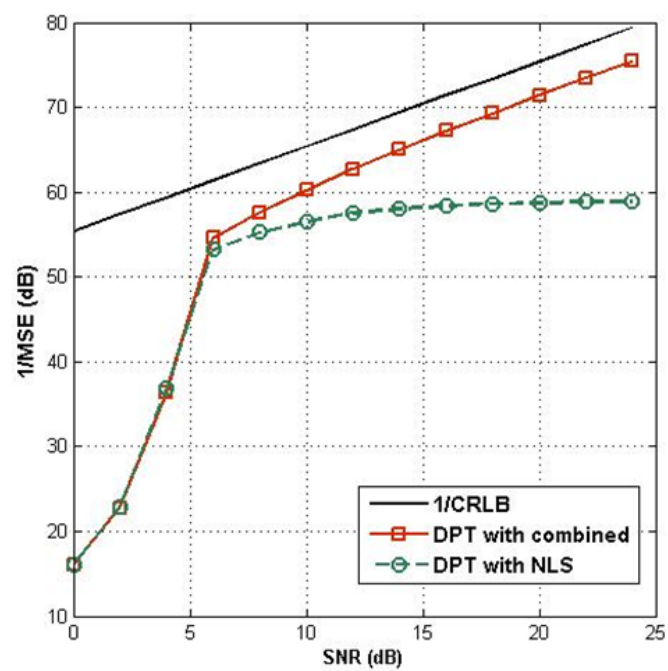

Fig. 5. MSE inverse versus SNR for chirp rate estimation of the LFM signal, using DPT with NLS and DPT with combined methods.

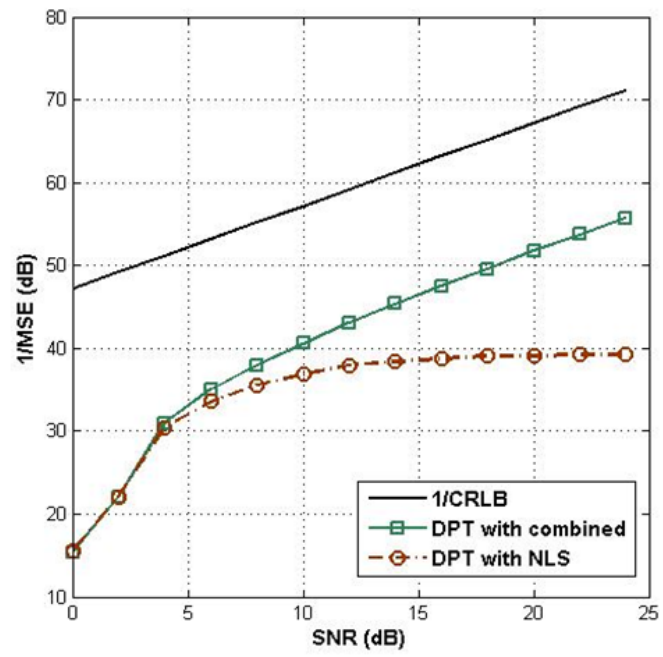

Fig. 6. MSE inverse versus SNR for central frequency estimation of the LFM signal, using DPT with NLS and DPT with combined methods.

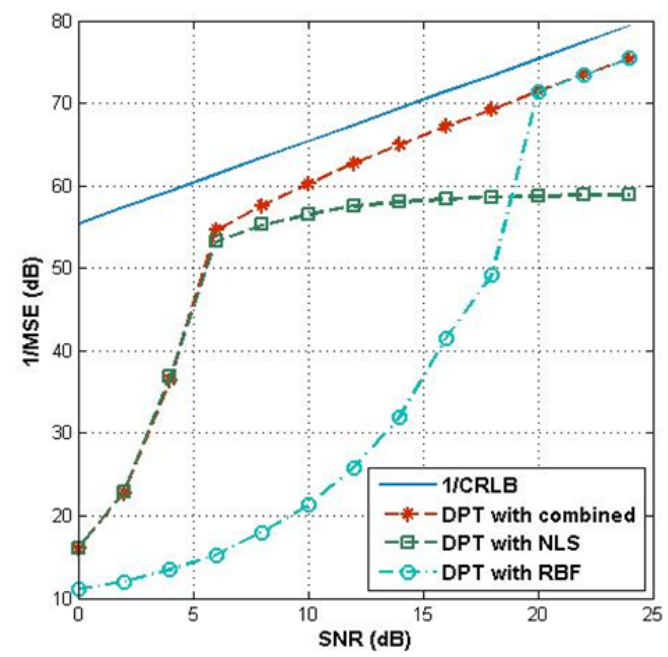

Fig. 7. MSE inverse versus SNR for chirp rate estimation of the LFM signal using DPT with NLS,DPT with RBF and DPT with combined methods.

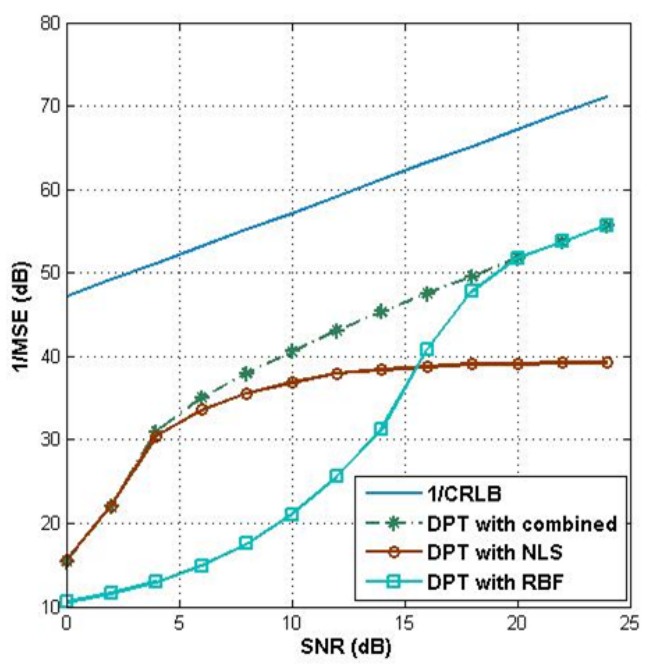

Fig. 8. MSE inverse versus SNR for central frequency estimation of the LFM signal, using DPT with NLS, DPT with RBF and DPT with combined methods. 
the FFT length of the NLS is 256. In Fig. 4, MSE inverse versus SNR of the central frequency estimation is illustrated for two methods (DPT with NLS and DPT with $\mathrm{RBF}$ ). As can be seen, in estimation of central frequency and chirp rate, MSE inverse and the CRLB inverse in DPT with RBF method are closer than the DPT with NLS method in higher SNR. In Fig. 4, it is quite clear that the frequency estimation error decreases due to the high precision in the chirp rate estimation process.

Therefore, DPT with RBF method has a better performance in higher SNRs as compared to DPT with NLS. On the other hand, as it is clear from Figs. 5 and 6, compared to DPT with NLS method, MSE inverse in DPT with combined method has become closer to CRLB inverse and also in Fig. 6, the central frequency estimation error has decreased because of high precision in the chirp rate estimation in DPT with combined method. For better comparison, DPT method with all the three techniques are shown in Fig. 7and 8. In the lower SNRs, the DPT with combined method has better results compared to DPT with RBF. Also in this method, the MSE inverse are closer to the CRLB inverse.

\section{Computational Complexity}

For the RBF method, $k$ is number of steps and computations must be repeated for $k(4 N+8)$ times, while in periodogram it will be $1.5 N_{\mathrm{FFT}} \log _{2}\left(N_{\mathrm{FFT}}\right)+N_{\mathrm{FFT}}$ times, and as we know its accuracy increases for higher values of $N_{\text {FFT }}$. In each method, the complex vector of $\mathbf{r}(t)$ with the length of $N-\tau$, requires $3(N-\tau)$ times of multiplication and summation for each repeat. The combined technique uses the NLS in the case of single complex exponential with a small FFT length ( $\left.N_{\text {FFTsyn }}\right)$, and $k-k_{\text {FFTsyn }}$ steps according to the RBF method. Also, we consider the $k_{\mathrm{FFTsyn}}$ value to be $N_{\text {FFT_syn }}=2^{k_{\text {FFTsyn }}} . N$ is the number of input samples. Table 1 shows the computational load of DPT method with NLS, RBF and combined technique for $N=12$, $N_{\text {FFT }}=256, k_{\text {syn }}=11$ and $N_{\text {FFTsyn }}=32$ as follows.

\begin{tabular}{|c|c|c|}
\hline Method & $\begin{array}{c}\text { The method } \\
\text { parameters }\end{array}$ & $\begin{array}{c}\text { The number of complex } \\
\text { multiplication and summation }\end{array}$ \\
\hline $\begin{array}{c}\text { DPT with NLS } \\
\text { (periodogram } \\
\text { based on FFT) }\end{array}$ & $\begin{array}{c}N=12 \\
N_{\mathrm{FFT}}=256\end{array}$ & $\begin{array}{c}1.5 N_{\mathrm{FFT}} \log _{2}\left(N_{\mathrm{FFT}}\right)+ \\
N_{\mathrm{FFT}}+3(N-1)=3361\end{array}$ \\
\hline DPT with RBF & $\begin{array}{c}N=12 \\
k=16\end{array}$ & $\begin{array}{c}k(4 N+8)+3(N-1) \\
=929\end{array}$ \\
\hline & $\begin{array}{c}N=12 \\
k_{\mathrm{syn}}=11 \\
\text { DPT with }\end{array}$ & $\begin{array}{c}k_{\mathrm{syn}}(4 N+8)+ \\
1.5 N_{\mathrm{FFT} \_ \text {syn }} \log _{2} N_{\mathrm{FFT} \_ \text {syn }} \\
+N_{\text {FF__syn }}+3(N-1)=921\end{array}$ \\
\hline
\end{tabular}

Tab. 1. Computational load of DPT with NLS (based on FFT), DPT with RBF and DPT with combined methods for chirp rate estimation.

\section{Conclusions}

This paper proposes and uses a novel combined technique in order to increase precision of estimation of the phase parameters of the LFM signal in DPT method. The combined technique attempts to estimate complex exponential frequency with less computations. The results of the simulations show that the error of the central frequency estimation has decreased due to the higher accuracy in the estimation of chirp rates using DPT with RBF and DPT with combined methods (compared to DPT with NLS). Also, it can be seen that in lower SNRs, the DPT with combined technique has better results than DPT with RBF, and MSE inverse are closer to CRLB inverse. And according to the computational table, the DPT with RBF and DPT with combined methods have less computational load than the DPT with NLS (periodogram based on FFT) method.

\section{References}

[1] CHAN, Y. K., CHUA, M. Y., KOO, V. C. Side lobe reduction using simple two and tri stages non-linear frequency modulation (NLFM). Progress in Electromagnetic Research, 2009, vol. 98, p. 33-52. DOI: 10.2528/PIER09073004

[2] ATKINS, P. R., COLlins, T., FOOTE, K. G. Transmit-signal design and processing strategies for sonar target phase measurement. IEEE Journal of Selected Topics in Signal Processing, 2007, vol. 1, no. 1, p. 91-104. DOI: 10.1109/JSTSP.2007.897051

[3] XING, M.,WU, R., LI, Y., et al. New ISAR imaging algorithm based on modified Wigner-Ville distribution. IET Radar, Sonar and Navigation, 2009, vol. 3, no. 1, p. 70-80. DOI: 10.1049/ietrsn:20080003

[4] LV, X. L., XING, M. D., WAN, C. R., et al. ISAR imaging of maneuvering targets based on the range centroid Doppler technique. IEEE Transactions on Image Processing, 2010, vol. 19, no. 1, p. 141-153. DOI: 10.1109/TIP.2009.2032892

[5] MARTONE, M. A multicarrier system based on the fractional Fourier transform for time-frequency-selective channels. IEEE Transactions on Communications, 2001, vol. 49, no. 6, p. 1011-1020. DOI: $10.1109 / 26.930631$

[6] MISARIDIS, T., JENSEN, J. A. Use of modulated excitation signals in medical ultrasound. Part I: Basic concepts and expected benefits. IEEE Transactions on Ultrasonics, Ferroelectrics, and Frequency Control, 2005, vol. 52, no. 2, p. 177-191. DOI: 10.1109/TUFFC.2005.1406545

[7] XIA, Y. Synthetic aperture radar interferometry. Chapter in Sciences of Geodesy-I. Ed. XU, G. Springer Berlin Heidelberg, 2010, p. 415-474. DOI: 10.1007/978-3-642-11741-1

[8] CUMMING, I. G., WONG, F. H. Digital Processing of Synthetic Aperture Radar Data. Norwood: Artech House, 2005. ISBN-13: 978-1580530583

[9] BARBAROSSA, S. Detection and imaging of moving objects with synthetic aperture radar. Part 1: Optimal detection and parameter estimation theory. IEE Proceedings F: Radar and Signal Processing, 1992, vol. 139, no. 1, p. 79-88. DOI: 10.1049/ip-f2.1992 .0010

[10] YANG, L., ZHAO, L., BI , G., ZHANG, L. SAR ground moving target imaging algorithm based on parametric and dynamic sparse 
Bayesian learning. IEEE Transactions on Geosciences and Remote Sensing, 2016, vol. 54, no. 4, p. 2254-2267. DOI: 10.1109/TGRS.2015.2498158

[11] Barbarossa, S., Di LORENZO, P., VecChiarelli, P. Parameter estimation of 2D multi-component polynomial phase signals: An application to SAR-imaging of moving targets. IEEE Transactions on Signal Processing, 2014, vol. 62, no. 17, p. 4375-4389. DOI: 10.1109/TSP.2014.2333553

[12] ABATZOGLOU, T. J. Fast maximum likelihood joint estimation of frequency and frequency rate. In IEEE International Conference on Acoustics, Speech, and Signal Processing (ICASSP '86). Tokyo (Japan), 1986, vol. 11, p. 1409-1412. DOI: 10.1109/ICASSP.1986.1168717

[13] O'SHEA, P. Improving polynomial phase parameter estimation by using nonuniformly spaced signal sample method. IEEE Transactions on Signal Processing, 2012, vol. 60, no. 7, p. 3405-3414. DOI: $10.1109 /$ TSP.2012.2191546

[14] DJURIC, P. M., KAY, S. M. Parameter estimation of chirp signals. IEEE Transactions on Acoustics, Speech, and Signal Processing, 1990 , vol. 38 , no. 12 , p. 2118-2126. DOI: $10.1109 / 29.61538$

[15] KUMARESAN, R., VERMA, S. On estimating the parameters of chirp signals using rank reduction techniques. In Proceedings of the 21st Asilomar Conference on Signals, Systems and Computers. 1987, p. 555-558.

[16] SHAMSUNDER, S., GIANNAKIS, G. B., FRIEDLANDER, B. Estimating random amplitude polynomial phase signals: A cyclostationary approach. IEEE Transactions on Signal Processing, 1995, vol. 43, no. 2, p. 492-505. DOI $10.1109 / 78.348131$

[17] ZHENG, J., REN, A. F., SU, T., et al. An algorithm for phase parameters estimation of multi-chirp signals. Journal of Xi'an Jiaotong University, 2013, vol. 47, no. 2, p. 69-74. (In Chinese)

[18] PELEG, S., PORAT, B. Estimation and classification of polynomial-phase signals. IEEE Transactions on Information Theory, 1991, vol. 37, no. 2, p. 422-430. DOI: 10.1109/18.75269

[19] PELEG, S., FRIEDLANDER, B. The discrete polynomial-phase transform. IEEE Transactions on Signal Processing, 1995, vol. 43, no. 8 , p. 1901-1914. DOI: $10.1109 / 78.403349$

[20] KAY, S. Signal fitting with uncertain basis functions. IEEE Signal Processing Letters, 2001, vol. 6, no. 18, p. 383-386. DOI: 10.1109/LSP.2011.2140397

[21] KAY, S. M. Fundamentals of Signal Processing-Estimation Theory. Englandwood Cliffs. NJ: Prentice Hall, 1993. ISBN-13: 978-0133457117

[22] KAY, S. M. Fundamentals of Statistical Signal Processing: Practical Algorithm Development. Vol. 3. $1^{\text {st }}$ ed. Prentice Hall, 2013. ISBN-13: 978-0132808033
[23] KAY, S. A computationally efficient nonlinear least squares method using random basis functions. IEEE Signal Processing Letters, 2013, vol. 20, no. 7, p. 721-724. DOI: 10.1109/LSP.2013.2264808

[24] STOICA, P., MOSES, R. L. Spectral Analysis of Signals. $1^{\text {st }}$ ed. Upper Saddle River (NJ): Prentice Hall, 2005. ISBN: 0131139568

\section{About the Authors ...}

Nooshin RABIEE received her B.Sc. and M.Sc.degree in Electrical Telecommunication from the Islamic Azad University of Bushehr in 2009 and 2012. Now she is a Ph.D. student of the Islamic Azad University, Shiraz. She has been as a lecturer in the Islamic Azad University of Bushehr from 2012. Her research interests are signal processing, image processing, radar recognition.

Hamid AZAD (corresponding author) received the M.Sc. and Ph.D. degrees both in Electerical Engineering in 2007 and 2013 from Shiraz University, Shiraz, Iran. He was with the Islamic Azad University, Science and Research Branch, Fars, Iran from 2011 to 2014 and has been with the Islamic Azad University, Shiraz Branch from 2014 till now as an assistant professor. He published more than 20 referable papers in international journals and conferences. His main research interests are statistical signal processing, estimation and detection theory and also sparse signal processing.

Naser PARHIZGAR received the M.Sc. degree in Electrical Telecommunication from the Islamic Azad University of Najaf abad in 2007 and the Ph.D. degree in Electrical Telecommunication Engineering from the Islamic Azad University at Science \& Research Branch, Tehran, Iran in 2013. Total projects have been done by him, is more than 30 . He has published more than 60 referable papers in international journals and conferences. From 1992 to 2007, he was with the Iran Electronics Industries. He has been a Dean of Engineering College of Islamic Azad University at Science and Research Branch, Fars, Iran from 2010 to 2012. He has been a Head of Telecommunications group from 2009 to 2010. He is an Assistant Professor of Electrical Engineering of Shiraz Branch, Islamic Azad University, Shiraz, Iran. His research interests are signal processing, radar system, wireless communications, antenna, radio networks, image processing. 